\title{
FENESTRATION OF THE BASILAR ARTERY IN A 13-YEAR-OLD BOY DETECTED IN MAGNETIC RESONANCE
}

Svjetlana MUJAGIĆ ${ }^{1}$, Nihad MEŠANOVIĆ

${ }^{1}$ Department of Radiology and Nuclear Medicine ${ }^{2}$ Sector for Information Technology, University Clinical Hospital Tuzla, Tuzla, Bosnia and Herzegovina

Control magnetic resonance (MR) imaging of the brain in a 13-year-old boy with epilepsy confirmed a non-expansive focal parenchymal lesion in the white matter of the left frontal lobe, coressponding to heterotopic grey
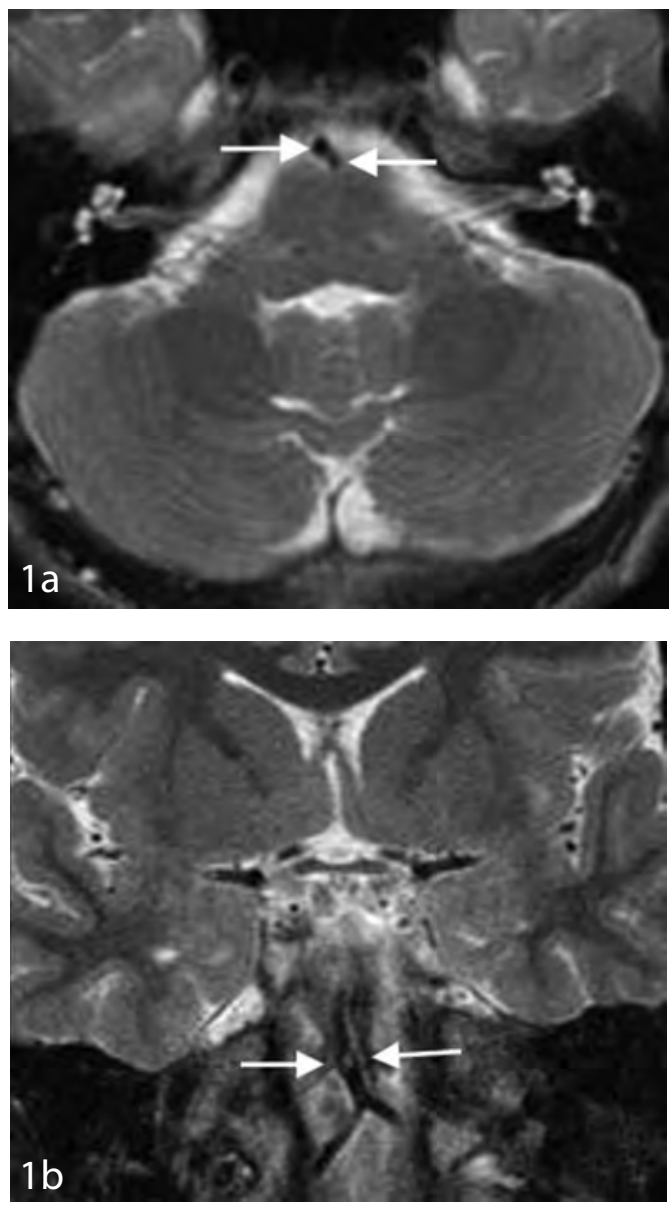

matter revealed two years before. MR scans did not show new lesions in the brain parenchyma. The axial T2-weighted image showed double flow voids (Fig. 1a), while the coronal T2-weighted image showed a small loss of signal intensity (Fig. 1b) in the proximal part of the basilar artery (BA), which wasn't detected on the first exam. MR angiography showed a type 3 (classification according to the location of fenestration introduced by Tanaka et al. in 2006), medium size, slit-like fenestration at the proximal segment of the BA, without evidence of aneurysm, thrombus or dissection (Fig. 2, 3). The fenestration window was 3.6 $\mathrm{mm}$; the right anterior inferior cerebellar artery (AICA) arose from the fenestrated trunk

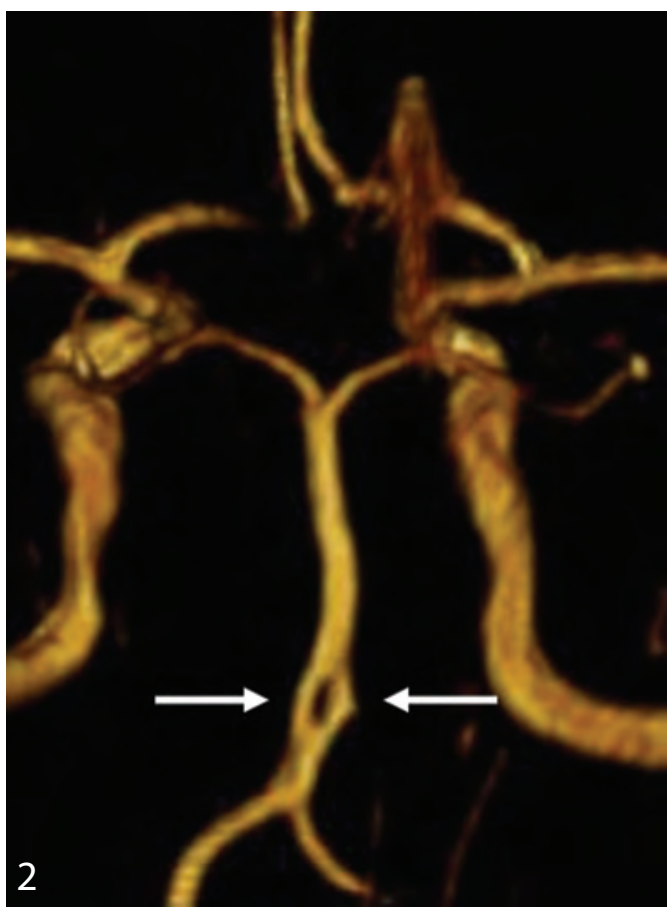




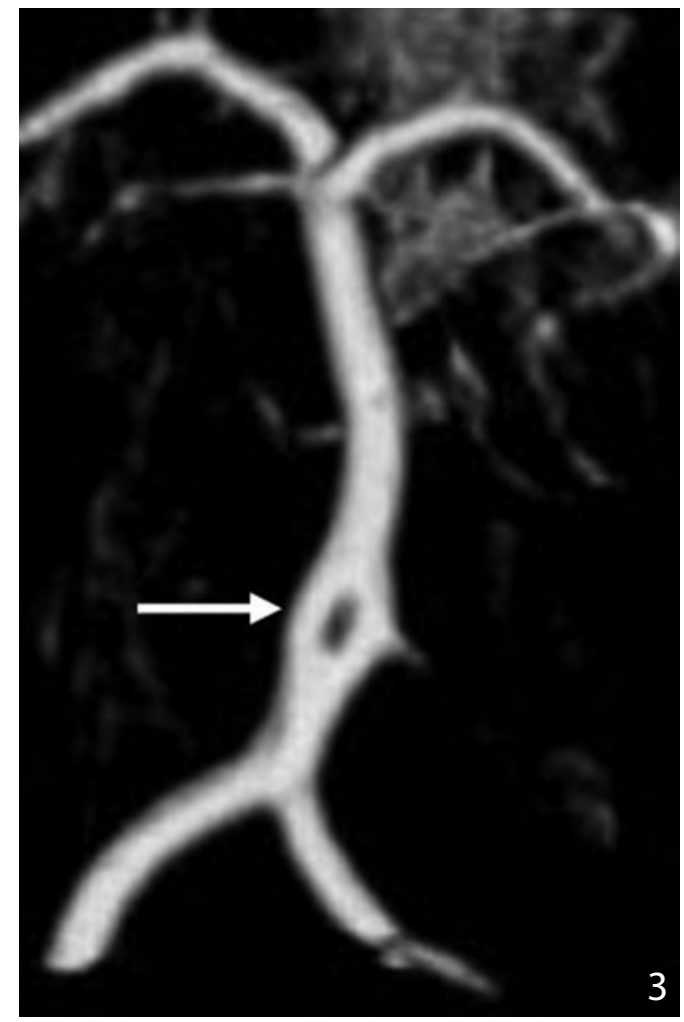

while the left AICA arose distal to the fenestrated trunk. The BA fenestration in this case was an incidental finding and wasn't related to the dysplasia or epilepsy. BA fenestration is rare anatomic variant of posterior cerebral circulation that can be associated with cerebral aneurysm and thrombosis. Most published literature on BA has focused on adults, while fenestration of the cerebral artery in children has rarely been published, although fenestration is a congenital anomaly whose incidence in children must be equal to its incidence in adults. Intracranial arterial fenestration can be missed even on good quality MRI study, and all children with neuroimaging should have their scans be carefully inspected for this anatomic variant.

Key words: Fenestration - Basilar artery.

Authors' contributions: Conception and design: SM; Acquisition, analysis and interpretation of data: SM, NM; Drafting the manuscript: SM; Revising it critically for important intellectual content: SM, NM.

Conflict of interest: The authors declare that they have no conflict of interest.

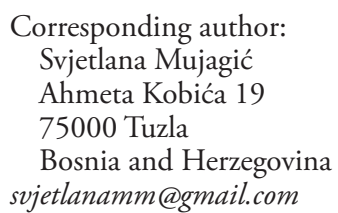

Tel.: + 38761661 080; Fax.: + 38735251456

Received: October 30, 2013; Accepted: December 9, 2014

Copyright (C) 2014 by University Clinical Hospital

Tuzla. E-mail for permission to publish:

paediatricstoday@ukctuzla.ba 